Provided for non-commercial research and education use. Not for reproduction, distribution or commercial use.

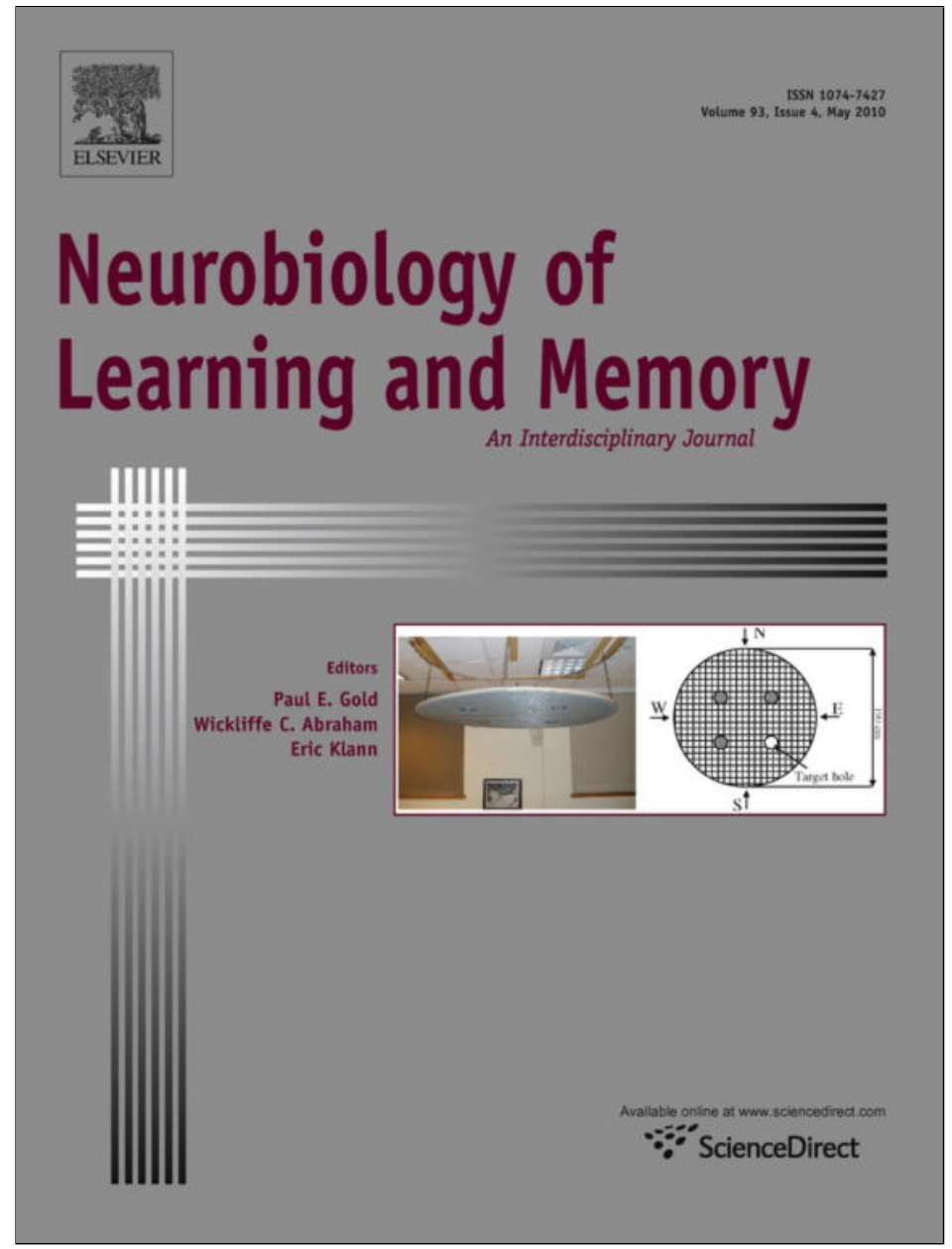

This article appeared in a journal published by Elsevier. The attached copy is furnished to the author for internal non-commercial research and education use, including for instruction at the authors institution and sharing with colleagues.

Other uses, including reproduction and distribution, or selling or licensing copies, or posting to personal, institutional or third party websites are prohibited.

In most cases authors are permitted to post their version of the article (e.g. in Word or Tex form) to their personal website or institutional repository. Authors requiring further information regarding Elsevier's archiving and manuscript policies are encouraged to visit:

http://www.elsevier.com/copyright 


\title{
Gender differences in the effects of post-learning emotion on consolidation of item memory and source memory
}

\author{
Bo Wang ${ }^{\mathrm{a}, \mathrm{b}}$, Xiaolan $\mathrm{Fu}^{\mathrm{a}, *}$ \\ ${ }^{a}$ State Key Laboratory of Brain and Cognitive Science, Institute of Psychology, Chinese Academy of Sciences, Beijing 100101, China \\ ${ }^{\mathrm{b}}$ Graduate University of Chinese Academy of Sciences, Beijing 100049, China
}

\section{A R T I C L E I N F O}

\section{Article history:}

Received 2 September 2009

Revised 31 January 2010

Accepted 24 February 2010

Available online 1 March 2010

\section{Keywords:}

Gender differences

Post-learning emotion

Item memory

Source memory

Consolidation

\begin{abstract}
A B S T R A C T
Item memory and source memory are two integral elements of episodic memory. Although many studies have examined the effect of emotion on item memory, little research has simultaneously taken into account item memory and source memory. In addition, in the majority of previous studies, learning stimuli are used as the source of emotion, making it difficult to understand whether emotion has an effect on encoding or on consolidation of episodic memory. Furthermore, although gender differences exist in neurophysiological responses to emotional stimuli, in many studies gender differences were neglected and this leaves the picture incomplete regarding the effect of emotion on episodic memory. In this study, we examined gender differences in the effects of post-learning emotion on consolidation of item memory and source memory. Participants learned neutral Chinese nouns, took a memory pretest, and were then randomly assigned to three conditions, in which they either watched a 3-min negative video clip, or watched a 3-min positive video clip, or remained calm and relaxed for $3 \mathrm{~min}$. Thirty minutes after the initial learning, participants took a memory posttest. We found that: (1) For females, post-learning negative emotion enhanced consolidation of item memory; however, neither negative emotion nor positive emotion had a significant effect on consolidation of source memory; (2) For males, neither negative nor positive emotion after learning had a significant effect on either item memory or source memory. Possible reasons for the gender differences, as well as the theoretical significance and practical implications of this study were discussed.
\end{abstract}

(c) 2010 Elsevier Inc. All rights reserved.

\section{Introduction}

People tend to have more vivid episodic memory for the events that arouse emotions (Bradley, Greenwald, Petry, \& Lang, 1992; Brown \& Kulik, 1977; Kensinger \& Corkin, 2003; LaBar \& Cabeza, 2006). Many lab studies have shown that emotion has an enhancing effect on episodic memory, by using either words (Anderson \& Shimamura, 2005; Bradley et al., 1992; Doerksen \& Shimamura, 2001; Kensinger \& Corkin, 2003; Kensinger \& Schacter, 2006) or pictures (Comblain, D’Argembeau, Van der Linden, \& Aldenhoff, 2004; D’Argembeau \& Van der Linden, 2005; Mather \& Nesmith, 2008 ) as the learning stimuli. However, some studies failed to find enhancement effect of emotion on episodic memory (Cook, Hicks, \& Marsh, 2007; Kapucu, Rotello, Ready, \& Seidl, 2008; Maddock \& Frein, 2009; Maratos, Allan, \& Rugg, 2000).

The majority of empirical studies, however, used a paradigm in which the source of emotion comes from the to-be-remembered

Project supported in part by grant from 973 Program (No. 2006CB303101) and the National Natural Science Foundation of China (No. 90820305).

* Corresponding author. Address: Institute of Psychology, Chinese Academy of Sciences, 4A Datun Road, Chaoyang District, Beijing 100101, China. Fax: +86 10 64872070 .

E-mail address: fuxl@psych.ac.cn (X. Fu). stimuli. The two inherent drawbacks of such a paradigm are that it is difficult to match stimuli for different conditions of emotion and that it is unclear whether the effect of emotion is on attention, encoding or consolidation, or a combination of them.

However, several recent studies have adopted a paradigm in which emotion is manipulated after learning (Anderson, Wais, John, \& Gabrieli, 2006; Liu, Graham, \& Zorawski, 2008; Nielson \& Lorber, 2009; Nielson \& Powless, 2007; Nielson, Yee, \& Erickson, 2005), excluding the possibility that emotion has any effect on encoding and providing a way to clarifying the role of emotion in memory consolidation. In a study of Nielson et al. (2005), participants learned a list of neutral words and then were assigned to watch a video clip that was either neutral or negatively arousing. Memory tests demonstrated that participants who watched a negatively arousing video clip remembered significantly more words than those who watched a neutral video clip, although the two groups had comparable pretest memory performance prior to watching the video clips. In a further study, Nielson and Powless (2007) examined the time-dependency of the effect of post-learning emotional arousal on memory consolidation. Participants learned a list of neutral words and then watched a positively or a negatively arousing video clip after delays of $0,10,30$ or $45 \mathrm{~min}$. The finding was that emotional arousal, whether positive or nega- 
tive, caused equivalent pleasure increase induced up to $30 \mathrm{~min}$ after learning, enhanced item memory consolidation.

In the above studies, the learning stimuli were neutral in emotionality. What if the learning stimuli are emotionally arousing? Nielson and Lorber (2009) addressed this question in a recent study. Participants learned four types of words, which ranged from high to low respectively in the dimensions of arousal and valence (i.e. words with high arousal and high valence, words with high arousal and low valence, words with low arousal and low valence, and words with low arousal and high valence). Ten minutes after the learning, participants viewed either a positive or a neutral video clip. Memory test conducted one week after the learning indicated that post-learning emotional arousal significantly enhanced memory for words. Importantly, no interaction was found between the emotionality of learned words and the post-learning emotional arousal, meaning that the memory for all the aforementioned four types of words was enhanced.

In summary, the above studies demonstrated that emotion induced after learning could enhance consolidation of item memory. As we know, however, episodic memory consists of two integral elements: item memory and source memory. Source memory refers to recollection or recall of the context from which an item or fact is acquired, whereas item memory refers to recognition or recall of previously presented information itself. It is still unclear whether the enhancement effect of post-learning emotion can extend to source memory. To have a complete appreciation of how post-learning emotion affects episodic memory, it is necessary to investigate the effect of emotion on source memory.

Another aspect ignored by previous studies is gender differences. Some neuroimaging and electrophysilogical studies have showed that gender differences exist in the processing and retrieval of emotional stimuli. Cahill et al. (2001) found that in responses to emotional films, enhanced activity of the right, but not the left, amygdala in males was related to enhanced memory. On the contrary, for females enhanced activity of the left, not the right amygdala was related with enhanced memory. In addition, the effect of emotion on the left amygdala in females seemed to be more anterior, and to include more of the "extended amygdala" regions, than did the effect of emotion on the right amygdala in males. Canli, Desmond, Zhao, and Gabrieli (2002) found that in retrieval of emotional pictures, in comparison to females, males demonstrated more activation in a network including the right amygdala. In addition, females had significantly more brain regions where activation correlated with both ongoing evaluation of emotional experience and with subsequent memory for the most emotionally arousing pictures. Canli et al. (2002) suggested that such greater overlap in brain regions may explain the greater benefit in emotional memory enjoyed by females. More recently, Gasbarri et al. (2007) obtained electrophysiological evidence. Negative pictures elicited more robust P300 in the left hemisphere in females than in males, yet elicited a stronger P300 component in the right hemisphere in males than in females. Despite the above evidence, few studies so far have taken into account gender differences in studying the effect of post-learning emotion on episodic memory.

Considering the above research background, we aimed to address the issue of gender differences in the effect of post-learning emotion on both item memory and source memory. Participants viewed Chinese nouns in a font color of blue or red, remembering both the nouns and their font colors. Immediately after the learning, they undertook a pretest, which examined memory for both the nouns (item memory) and their font colors (source memory). Following the pretest, participants were then randomly assigned to three conditions, in which they watched a 3-min negative video clip, or watched a 3-min positive video clip, or remained calm and relaxed for three minutes. Then they stayed in the lab until a posttest that took place thirty minutes after the beginning of learning.
Based on the studies showing the enhancing effect of post-learning emotion on item memory (Nielson \& Lorber, 2009; Nielson \& Powless, 2007; Nielson et al., 2005), and the modulatory role of the amygdala in consolidation of memory in other brain regions including the hippocampus (McGaugh, 2002), we hypothesized that postlearning would enhance the consolidation of item memory. Meanwhile, considering the finding that post-learning emotion only enhanced the memory for gist rather than details of to-be remembered stimuli (Adolphs, Denburg, \& Tranel, 2001; Liu et al., 2008; Zorawski \& Killcross, 2003), and that source memory tasks can be used to examine memory for details (Kensinger \& Corkin, 2003), we expected that source memory would not be influenced by post-learning emotion. In addition, we hypothesized that females would be more likely than males to have better consolidation of item memory by watching a negative video. This hypothesis is based on the following findings: (1) Negative stimuli elicit more robust electrophysiological response in the left hemisphere in females than in males (Gasbarri et al., 2007); (2) Enhanced activity of the left amygdala in females was related to enhanced memory (Cahill et al., 2001). Furthermore, we hypothesized that females would also be more likely to have item memory benefit by watching a positive video, based on the following findings from previous research: (1) Females had greater overlap in brain regions in retrieval of emotional pictures (Canli et al., 2002); (2) Females were more emotionally reactive than males, and smiled more while reliving happiness and love (Chentsova-Dutton \& Tsai, 2007).

\section{Experiment for selection of video clips}

\subsection{Participants}

Forty-five participants (23 male and 22 female; mean age $=22.86$ years, $\mathrm{SD}=1.88$ years) were recruited to rate positive video clips. Forty-eight participants ( 24 male and 24 female; mean age $=23.20$ years, $\mathrm{SD}=2.44$ years) were recruited to rate negative video clips, in a procedure identical to that for the rating of positive video clips. The experiment was approved by the Institutional Review Board of the Institute of Psychology, Chinese Academy of Sciences.

\subsection{Materials}

In this study we needed a positive video clip and a negative video clip. In order to enhance the possibility of eventually coming up with two appropriate video clips, we prepared three positive video clips, respectively entitled No Lacking Money (positive 1), Dream Community (positive 2), and Olympic Torchbearer (positive 3). We also prepared three negative video clips: Wenchuan Earthquake (negative 1), Dull Ice Flower (negative 2) and Titanic (negative 3). All video clips lasted for $3 \mathrm{~min}$.

\subsection{Procedure}

Before watching a video clip, participants first reported their current pleasure and arousal on a 9-point Likert-type scale ranging from 0 to 8 . Immediately after the viewing, they again reported their current pleasure and arousal and filled in a questionnaire selected from Handbook of Emotion Elicitation and Assessment (Coan \& Allen, 2007). Each participant was asked to watch all the three video clips, with the order of video presentation balanced across participants.

\subsection{Results}

Descriptive data for the six video clips were given in Tables 1 and 2. Increase ratio was calculated by the formula: pleasure in- 
crease ratio $=($ pleasure $2-$ pleasure 1$) /$ pleasure 1 , and arousal increase ratio $=($ arousal $2-$ arousal 1$) /$ arousal 1 , where pleasure 1 and arousal 1 respectively stands for pleasure and arousal immediately before watching a video clip, and pleasure 2 and arousal 2 respectively stands for pleasure and arousal immediately after watching a video clip.

The analysis showed that positive 1 and positive 3 caused equivalent pleasure increase $(p=.805)$, but they caused significantly greater pleasure increase than positive $2(p=.01$ and $p=.019$, respectively). As to arousal increase, no significant difference existed between the three positive video clips (all $p s>.05$ ).

Negative 1 and negative 3 caused significantly greater pleasure decrease than negative 2 ( $p<.001$ and $p=.039$, respectively). Negative 1 caused significantly greater decrease than negative 3 $(p=.023)$. Regarding arousal increase, negative 1 and negative 3 caused significantly greater arousal increase than negative 2 $(p=.001$ and $p=.008$, respectively). However, negative 1 and negative 3 caused equivalent arousal increase $(p=.487)$.

We also conducted a repeated-measures ANOVA, with time 1 (before watching a video) and time 2 (after watching a video) being the two levels. The results demonstrated that for all the three positive videos, both pleasure and arousal at time 2 was significantly greater than at time 1 (all $p \mathrm{~s}<.001$ ). For all the three negative videos, pleasure at time 2 was significantly less than at time 1 $(p<.001, p=.001$ and $p<.001$ respectively).

A repeated-measures ANOVA indicated that for the videos negative 1 and negative 3 , arousal at time 2 was significantly greater than at time 1 (both $p s<.001$ ); for the video negative 2, however, arousal at time 2 did not significantly differ from arousal at time $1(p=.183)$.

Because some participants reported negative opinions towards an actor in positive 1 and that the majority of participants reported positive 3 as the most amusing, we chose positive 3 to elicit positive emotion in the primary experiment. To elicit negative emotion, we chose negative 1 because the majority of participants reported it to be the most sadness-arousing. Analysis indicated that positive 3 and negative 1 caused equivalent ratio of increase in arousal $\left(F(1,65)=.279, p=.599, \eta^{2}=.004\right)$.

\section{Primary experiment}

\subsection{Participants}

One hundred and forty-eight participants ( 75 male and $73 \mathrm{fe}-$ male; mean age $=21.58$ years, $S D=2.00$ years) were recruited from several universities in Beijing, China. They reported having no emotional disorders or history of serious physical illnesses. They were paid 20 Yuan (RMB) for the participation. This research was approved by the Institutional Review Board of the Institute of Psychology, Chinese Academy of Sciences.

\subsection{Stimuli}

For this study we first selected a total of 333 Chinese nouns from Dictionary of Word Frequency of Modern Chinese (Compiled by Beijing Institute of Languages). Then the 333 words were divided into four sets, each of which were respectively rated on the dimensions of pleasantness, arousal, concreteness, and familiarity. Each of the four sets were rated respectively by 28 participants ( 16 male, 12 female), 23 participants ( 11 male, 12 female), 28 participants (13 male, 15 female), and 24 participants ( 12 male, $12 \mathrm{fe}-$ male). All the words were rated on a scale of $1-9$ in the dimensions of pleasantness, arousal, concreteness, and familiarity.

From the 333 rated words a total of 88 neutral nouns (pleasantness mean $=5.06, \mathrm{SD}=.27$; arousal mean $=4.93, \mathrm{SD}=.27$; concreteness mean $=6.50, \mathrm{SD}=.51$; familiarity mean $=4.95, \mathrm{SD}=.75$ ) were chosen to be the learning stimuli. The word frequency range of the selected nouns is from 0.0077 to 0.0839 . The 88 nouns were evenly divided into set $\mathrm{A}$ and set $\mathrm{B}$, matched in terms of pleasantness, arousal, concreteness, familiarity, and word frequency. The nouns in set A and set B were used, in a counterbalanced manner across the participants, as target items in the learning session and distractors in the testing session. Four other nouns ( 2 nouns at the beginning and 2 nouns at the end of the learning list) were used to buffer primacy and recency effects.

\subsection{Design and procedure}

We used a $2 \times 3$ between-subjects design, with gender (male and female) and condition (negative, positive, and control) being the two factors. Participants were randomly assigned to three conditions as follows. In the negative condition, 52 participants (26 male and 26 female) watched a 3-min negative video clip after learning; in the positive condition, 48 participants ( 24 male and 24 female) watched a 3-min positive video clip after learning; in the control condition, 48 participants ( 25 male and 23 female) remained calm and relaxed for three minutes after learning.

During the learning, participants sat about $50 \mathrm{~cm}$ in front of computer screens. In each trial a crosshair first appeared at the center of screen for $1 \mathrm{~s}$, followed by a Chinese noun with either

Table 1

Descriptive data regarding actual ratings of pleasure and arousal for the six rated video clips (mean \pm SEM).

\begin{tabular}{|c|c|c|c|c|c|c|}
\hline & \multicolumn{3}{|c|}{ Positive video clips } & \multicolumn{3}{|c|}{$\underline{\text { Negative video clips }}$} \\
\hline & Positive 1 & Positive 2 & Positive 3 & Negative 1 & Negative 2 & Negative 3 \\
\hline Pleasure 1 & $4.51 \pm 0.19$ & $4.56 \pm 0.22$ & $4.33 \pm 0.18$ & $3.36 \pm 1.33$ & $3.13 \pm 0.20$ & $3.47 \pm 0.16$ \\
\hline Arousal 1 & $3.89 \pm 0.26$ & $4.08 \pm 0.23$ & $4.02 \pm 0.23$ & $3.49 \pm 0.19$ & $3.89 \pm 0.18$ & $3.47 \pm 0.15$ \\
\hline Pleasure 2 & $6.93 \pm 0.15$ & $5.89 \pm 0.21$ & $6.73 \pm 0.11$ & $1.02 \pm 1.01$ & $2.21 \pm 0.20$ & $1.68 \pm 0.19$ \\
\hline Arousal 2 & $5.96 \pm 0.22$ & $5.52 \pm 0.18$ & $6.04 \pm 0.14$ & $5.33 \pm 0.22$ & $4.41 \pm 0.22$ & $5.26 \pm 0.24$ \\
\hline
\end{tabular}

Note: Pleasure 1 and arousal 1 respectively stands for pleasure and arousal immediately before watching a video clip. Pleasure 2 and arousal 2 respectively stands for pleasure and arousal immediately after watching a video clip.

Table 2

Descriptive data regarding change of pleasure and arousal for the six rated video clips (mean \pm SEM).

\begin{tabular}{|c|c|c|c|c|c|c|}
\hline \multirow[t]{2}{*}{ Increase ratio } & \multicolumn{3}{|c|}{ Positive video clips (\%) } & \multicolumn{3}{|c|}{ Negative video clips (\%) } \\
\hline & Positive 1 & Positive 2 & Positive 3 & Negative 1 & Negative 2 & Negative 3 \\
\hline Pleasure & $65.0 \pm 14.0$ & $29.0 \pm 6.4$ & $56.0 \pm 5.9$ & $-70.6 \pm 4.3$ & $-17.8 \pm 10.3$ & $-42.8 \pm 9.5$ \\
\hline Arousal & $89.0 \pm 19.0$ & $60.0 \pm 18.0$ & $61.0 \pm 13.0$ & $82.4 \pm 16.6$ & $21.6 \pm 9.7$ & $66.7 \pm 11.2$ \\
\hline
\end{tabular}


red or blue font (Courier New, font size $=40$ ) appearing for $4 \mathrm{~s}$, against a white screen background. Participants were asked to use whatever mnemonics they thought were effective to remember both the nouns and their font colors. The learning phase lasted for $4 \mathrm{~min}$.

During the pretest immediately following the learning, 22 old nouns in set A were randomly selected, and mixed with 22 new nouns that were randomly selected from set $B$. The 44 nouns were randomly presented at the center of screen in the font color of black (Courier New, font size $=40$ ), against a white screen background. Participants first determined whether a noun was old or new by clicking a corresponding button on the screen. When they decided that a noun was old, they then judged the color of the noun by clicking a corresponding button on the screen. Their responses were self-paced.

Immediately following the pretest, about 10 min after learning, participants reported their current pleasure and arousal on a 9point Likert-type scale, and then watched either a 3-min positive video, or watched a 3-min negative video, or remained calm and relaxed for $3 \mathrm{~min}$, depending on the random group assignment. No mention was made as to the type of video clip to be watched. During the 3-min viewing or resting period, the light in the lab was turned off. After the viewing, participants in all the three conditions reported their current pleasure and arousal and then remained calm and relaxed in their seats until the posttest.

During the posttest that started 30 min after the initial learning, the remaining 22 old nouns in set A and the remaining 22 new nouns in set $B$ were mixed and randomly presented to participants. The procedure in the posttest was the same as in the pretest.

Following the posttest, participants were asked whether they did any rehearsals during the rest period. Then they filled in a 12-item arousal predisposition questionnaire (Coren, 1988), which examines susceptibility to emotional arousal. They also filled in a 10-item emotion regulation questionnaire (Gross \& John, 2003), in which four items aimed to examine emotion suppression and six items aimed to examine emotion reappraisal.

\section{Results}

The data analyses were conducted using SPSS for Windows 13.0. The level of statistical significance was set to be 0.05 .

\subsection{The effectiveness of emotion elicitation}

Repeated measures analysis was separately conducted on ratings of pleasure and arousal. The within-subjects factor was time (time 1: immediately before watching a video clip or taking a 3min rest; time 2 : immediately after watching a video clip or taking a 3-min rest). The between-subjects factors were condition (negative, neutral, and positive) and gender (male, female).

\subsubsection{Results based on ratings of pleasure}

The ANOVA analysis showed that there was no significant main effect of time $\left(F(1,137)=.496, p=.482, \eta^{2}=.004\right)$, indicating that pleasure at time 1 was equivalent to pleasure at time 2 . There was a significant main effect of condition $(F(2,137)=38.726$, $\left.p<.001, \eta^{2}=.361\right)$. Pleasure in the negative condition was significantly less than in both the positive condition $(p<.001)$ and the control condition $(p<.001)$; pleasure in the positive condition was significantly greater than in the control condition $(p<.001)$. The main effect of gender was significant $(F(1,137)=5.727$, $\left.p=.018, \eta^{2}=.04\right)$, indicating that males had greater pleasure than did females.

Most importantly, there was a significant interaction between time and condition $\left(F(2,137)=67.648, p<.001, \eta^{2}=.497\right.$ ) (see
Fig. 1), indicating that at time 1 participants across the three groups had comparable pleasure $(F(2,140)=.563, p=.571$, $\eta^{2}=.008$ ); however, at time 2 participants in the positive condition had significantly greater pleasure than both the participants in the control condition $(p<0.001)$ and in the negative condition $(p<0.001)$. In addition, at time 2 participants in the negative condition had significantly less pleasure than in the control condition $(p<0.001)$. There were no other significant interactions (time $\times$ gender, $F(1,137)=.003, p=.953, \eta^{2}<.001$; gender $\times$ condition, $F(2,137)=1.364, p=.259, \eta^{2}=.02$; time $\times$ gender $\times$ condition, $\left.F(1,137)=.933, p=.013, \eta^{2}=.013\right)$.

The above results offered the evidence that the positive and negative video clips were effective in enhancing or reducing pleasure in both male and female participants as we expected.

\subsubsection{Results based on ratings of arousal}

Due to a technical problem, the ratings of arousal of four participants were not collected. The ANOVA analysis showed that there was a significant main effect of time $(F(1,133)=13.621, p<.001$, $\eta^{2}=.093$ ), indicating that arousal at time 2 was significantly greater than at time 1 . There was also a significant main effect of condition $\left(F(2,133)=6.506, p=.002, \eta^{2}=.089\right)$, indicating that arousal in the positive condition was significantly greater than in the control condition $(p=.001)$ and in the negative condition $(p=.033)$. Arousal in the negative condition, however, did not significantly differ from that in the control condition $(p=.15)$.

There was a significant interaction between time and condition $\left(F(2,133)=10.837, p<.001, \eta^{2}=.14\right)$ (see Fig. 2$)$, suggesting that at time 1 participants across the three conditions had comparable arousal $\left(F(2,133)=.017, p=.983, \eta^{2}<.001\right)$. However, at time 2 participants both in the positive condition and in the negative condition had significantly greater arousal than in the control condition ( $p<.001$ and $p=0.02$ respectively). Participants in the positive condition had significantly greater arousal than in the negative condition $(p=.001)$. The main effect of gender was nonsignificant $\left(F(1,133)=2.125, p=.147, \eta^{2}=.016\right)$. No other significant interactions were found (time $\times$ gender, $F(1,133)=.219, p=.641$, $\eta^{2}=.002$; gender $\times$ condition, $F(2,133)=.796, p=.453, \eta^{2}=.012$; time $\times$ gender $\times$ condition, $\left.F(2,133)=.985, p=.376, \eta^{2}=.015\right)$.

The above results indicated that both male and female participants who watched one of the two video clips experienced significantly greater arousal than those in control condition, although the participants who watched the positive video clip experienced significantly greater arousal than those who watched the negative one.

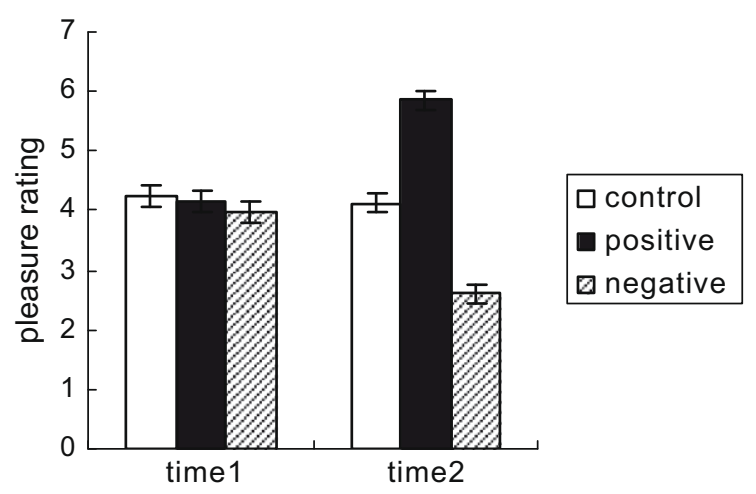

Fig. 1. Pleasure ratings (mean $\pm S E M$ ) as a function of time. At time 1 , there was no significant effect of condition $\left(F(2,140)=.56, p=.571, \eta^{2}=.008\right)$. At time 2 , the main effect of condition was significant $\left(F(2,140)=98.74, p<.001, \eta^{2}=.585\right)$, indicating that pleasure in negative condition was significantly less than in both positive condition $(p<.001)$ and control condition $(p<.001)$ and that pleasure in positive condition was significantly greater than in control condition $(p<.001)$. 


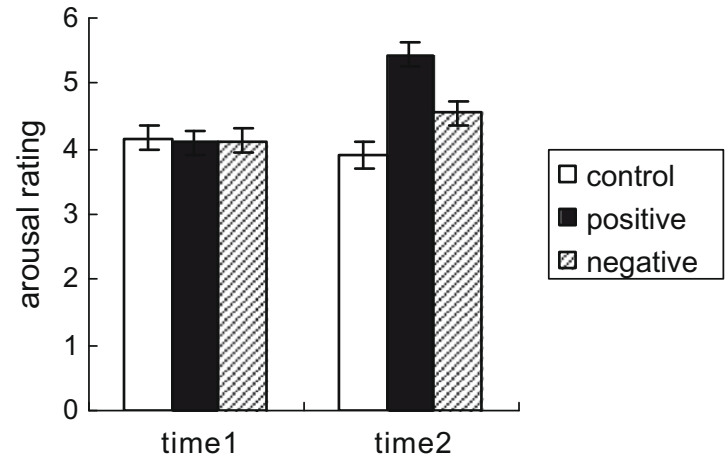

Fig. 2. Arousal ratings (mean \pm SEM) as a function of time. At time 1, participants in the three conditions had comparable arousal ratings $(F(2,136)=.022, p=.978$, $\left.\eta^{2}<.001\right)$. However, at time 2 , there was a significant main effect of condition $\left(F(2,140)=17.008, p<.001, \eta^{2}=.195\right)$, indicating that arousal both in positive condition and negative was significantly greater than in control condition $(p<.001$ and $p=.01$ respectively) and that arousal in positive condition was significantly greater than in negative condition $(p=.001)$.

In our study the videos were rated on a 9-point Likert scale, with 0 indicating least pleasure or arousal and 8 indicating greatest pleasure and arousal. In our opinion, under the condition that pleasure and arousal prior to watching a video do not significantly differ from 4 , a video can be considered as eliciting positive arousal if post-watching pleasure and arousal are both significantly greater than 4; a video can be considered as eliciting negative arousal if post-watching pleasure is significantly less than 4 and post-watching arousal is significantly greater than 4 . We conducted several one-sample $t$ tests and presented the results in Tables 3 and 4 . The results indicate that both the positive video and negative video used for the primary experiment were respectively effective in inducing pleasure and arousal as expected.

\subsection{The effect of post-learning emotion on item memory consolidation}

Univariate ANOVA was carried out with the between-subjects factors being gender (male and female) and condition (negative, positive, and control). The dependent measure of memory consol- idation was determined in terms of memory decrease. The item memory decrease was determined by subtracting $P r$ in the posttest from $\mathrm{Pr}$ in the pretest ( $\mathrm{Pr}=$ hit rates - false alarm rates). The data of 4 participants were excluded because their item memory was at chance level ( $P r$ values were lower than or equal to zero). The final statistical analyses were based on the data of 143 participants (71 female and 72 male). The data of one participant, whose item memory decrease was beyond three standard deviations of the group mean, was also excluded.

Descriptive data for item memory at the pretest and posttest are displayed in Table 5. Item memory decrease is defined as pretest score minus posttest score. The smaller the item memory decrease, the better the item memory consolidation.

Univariate ANOVA showed that participants across the three conditions had comparable pretest item memory performance $\left(F(2,137)=2.762, p=.067, \eta^{2}=.039\right)$. In addition, with regard to the pretest item memory, there was no significant main effect of gender $\left(F(1,137)=.949, p=.332, \eta^{2}=.007\right)$. The interaction between condition and gender was nonsignificant either $\left(F(2,137)=.549, p=.579, \eta^{2}=.008\right)$.

When the item memory decrease was the dependent measure, the analysis demonstrated that there was neither a significant main effect of condition $\left(F(2,137)=1.838, p=.163, \eta^{2}=.026\right)$ nor a significant main effect of gender $(F(1,137)=0.835, p=.362$, $\left.\eta^{2}=.006\right)$. However, there was a significant interaction between gender and condition $\left(F(1,137)=3.635, p=.029, \eta^{2}=.05\right.$ ) (see Fig. 3). Further analyses showed that the main effect of condition for males is nonsignificant $\left(F(2,69)=.69, p=.505, \eta^{2}=.02\right)$, indicating that males across the three conditions had comparable item memory decrease (all $p s>.05$ ). However, the main effect of condition for females is significant $\left(F(2,68)=4.266, p=.018, \eta^{2}=.11\right)$. Further analyses showed that females in the negative condition had significantly less item memory decrease than in the control condition $(p=.007)$ and than in the positive condition $(p=.039)$. No significant difference was observed between females in the positive condition and in the control condition $(p=.476)$.

In addition, we also calculated the $d^{\prime}$ value for each participant as the dependent measure. The analysis using $d^{\prime}$ demonstrated that there was neither a significant main effect of condition $\left(F(2,137)=1.698, p=.187, \eta^{2}=.024\right)$ nor a significant main effect

Table 3

$t$ test results for the positive video.

\begin{tabular}{|c|c|c|c|c|c|c|}
\hline & \multirow[t]{2}{*}{$t$} & \multirow[t]{2}{*}{ df } & \multirow[t]{2}{*}{ Sig. (2-tailed) } & \multirow[t]{2}{*}{ Mean difference } & \multicolumn{2}{|c|}{$95 \%$ Confidence interval of the differenc } \\
\hline & & & & & Lower & Upper \\
\hline Pleasure 1 & .784 & 47 & .437 & .146 & -.23 & .52 \\
\hline Arousal 1 & .552 & 47 & .584 & .104 & -.28 & .48 \\
\hline Pleasure 2 & 13.280 & 47 & $<.001$ & 1.854 & 1.57 & 2.14 \\
\hline Arousal 2 & 10.821 & 47 & $<.001$ & 1.438 & 1.17 & 1.70 \\
\hline
\end{tabular}

Note: Pleasure 1 and arousal 1 respectively stands for pleasure and arousal immediately before watching a video clip. Pleasure 2 and arousal 2 respectively stands for pleasure and arousal immediately after watching a video clip. The values of pleasure 1, arousal 1, pleasure 2 and arousal 2 are respectively compared with 4 by conducting one-sample $t$ tests.

Table 4

$t$ test results for the negative video.

\begin{tabular}{|c|c|c|c|c|c|c|}
\hline & \multirow[t]{2}{*}{$t$} & \multirow[t]{2}{*}{ df } & \multirow[t]{2}{*}{ Sig. (2-tailed) } & \multirow[t]{2}{*}{ Mean difference } & \multicolumn{2}{|c|}{$95 \%$ Confidence interval of the difference } \\
\hline & & & & & Lower & Upper \\
\hline Pleasure 1 & -.117 & 48 & .907 & -.020 & -.37 & .33 \\
\hline Arousal 1 & .651 & 46 & .519 & .128 & -.27 & .52 \\
\hline Pleasure 2 & -7.449 & 48 & $<.001$ & -1.388 & -1.76 & -1.01 \\
\hline Arousal 2 & 2.671 & 46 & .013 & .553 & .12 & .99 \\
\hline
\end{tabular}

Note: Pleasure 1 and arousal 1 respectively stands for pleasure and arousal immediately before watching a video clip. Pleasure 2 and arousal 2 respectively stands for pleasure and arousal immediately after watching a video clip. The values of pleasure 1, arousal 1, pleasure 2 and arousal 2 are respectively compared with 4 by conducting one-sample $t$ tests. 
Table 5

Item memory performance (mean \pm SEM).

\begin{tabular}{|c|c|c|c|c|c|c|}
\hline \multirow[t]{2}{*}{ Condition } & \multicolumn{3}{|l|}{ Male } & \multicolumn{3}{|l|}{ Female } \\
\hline & Pretest score & Posttest score & Memory decrease & Pretest score & Posttest score & Memory decrease \\
\hline Control & $0.5988 \pm 0.0386$ & $0.5059 \pm 0.0433$ & $0.0929 \pm 0.0275$ & $0.6680 \pm 0.0396$ & $0.4585 \pm 0.0408$ & $0.2095 \pm 0.0386$ \\
\hline Positive & $0.6136 \pm 0.0434$ & $0.4697 \pm 0.0452$ & $0.1440 \pm 0.0353$ & $0.6420 \pm 0.0286$ & $0.4697 \pm 0.0428$ & $0.1723 \pm 0.0364$ \\
\hline Negative & $0.5600 \pm 0.0312$ & $0.4273 \pm 0.0386$ & $0.1327 \pm 0.0320$ & $0.5511 \pm 0.0400$ & $0.4867 \pm 0.0346$ & $0.0644 \pm 0.0345$ \\
\hline
\end{tabular}

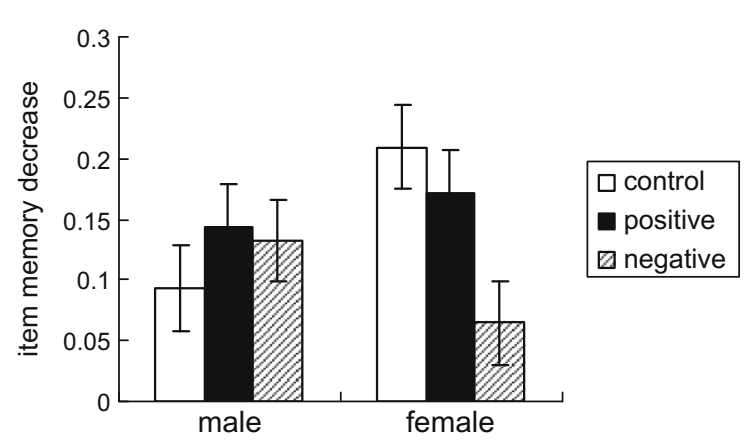

Fig. 3. Gender differences in the effect of post-learning emotion on decrease of item memory (bars are represented as mean \pm SEM). While males in the three conditions had equivalent item memory decrease $(p=.558)$, females in negative condition had significantly less decrease in item memory than in control condition $(p=.007$, Cohen's $d=.82$ ), but in positive condition had equivalent item memory decrease to that in control condition $(p=.24)$.

of gender $\left(F(1,137)=0.897, p=.345, \eta^{2}=.007\right)$. However, there was a significant interaction between gender and condition $\left(F(1,137)=5.231, p=.006, \eta^{2}=.071\right)$. Further analyses showed that the main effect of condition for males is nonsignificant $\left(F(2,69)=1.122, p=.332, \eta^{2}=.031\right)$, indicating that males across the three conditions had comparable item memory decrease (all $p s>.05)$. However, the main effect of condition for females was significant $\left(F(2,68)=4.621, p=.013, \eta^{2}=.12\right)$. Further analyses showed that females in the negative condition had significantly less item memory decrease than in the control condition $(p=.003)$. No significant difference was observed between females in the positive condition and in the control condition $(p=.138)$. No significant difference was found between the negative condition and positive condition $(p=.125)$. Therefore, when $d^{\prime}$ value were included as the dependent measure in the analyses, the results were in agreement with the results based on $\operatorname{Pr}$ values (hit rates minus false alarm rates) except that no significant difference was found between the negative condition and positive condition.

\subsection{The effect of emotion on source memory consolidation}

Source memory was calculated as the proportion of correctly recognized nouns whose font colors were correctly identified. Descriptive data for source memory at the pretest and posttest are displayed in Table 6 . Source memory decrease is defined as pretest score minus posttest score. The smaller the source memory decrease, the better the source memory consolidation.
Univariate ANOVA showed that participants across the three conditions had comparable pretest source memory performance $\left(F(2,137)=2.333, p=.101, \eta^{2}=.03\right)$. In addition, concerning the pretest source memory performance, no significant main effect of gender was found $\left(F(1,137)=.053, p=.818, \eta^{2}<.001\right)$. The interaction between condition and gender was nonsignificant, either $\left(F(2,137)=1.493, p=.228, \eta^{2}=.02\right)$.

Univariate ANOVA with the source memory decrease being the dependent measure demonstrated that there was neither a significant main effect of condition $\left(F(2,137)=1.351, p=.263, \eta^{2}=.02\right)$ nor a significant main effect of gender $(F(1,137)=.914, p=.341$, $\left.\eta^{2}=.01\right)$. In addition, the interaction between gender and condition was nonsignificant $\left(F(1,137)=.754, p=.472, \eta^{2}=.01\right)$ (see Fig. 4 ).

\subsection{The effects of individual differences}

The median scores of the measures of arousal predisposition, emotion reappraisal and emotion suppression were calculated. The median score of each of the three measures was used to split participants into two groups. Table 7 gives the number of participants in the low level and high level groups. Table 8 gives the range and median scores for arousal predisposition, emotion reappraisal and suppression.

Univariate ANOVA was conducted with the factors being using condition (negative, positive, and control) and emotion measure (high level and low level).

Due to a technical error, the data of nine participants were not collected (four participants on arousal predisposition, three participants on emotion reappraisal, and two participants on emotion suppression).

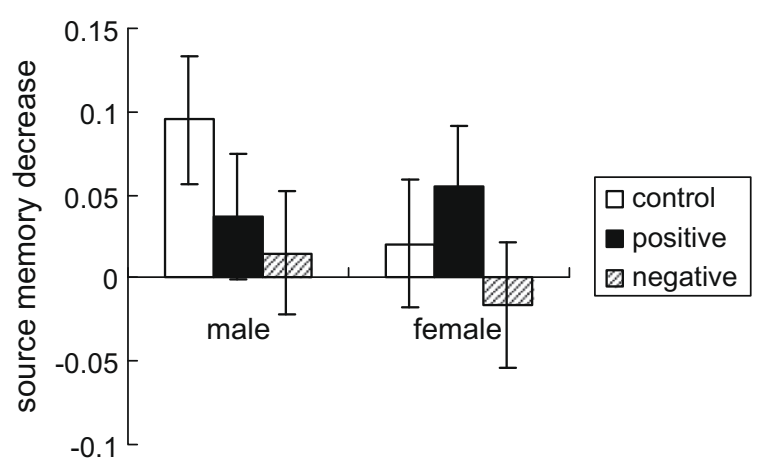

Fig. 4. For both males and females, source memory decrease in the negative and positive conditions did not significantly differ from that in control condition (bars are represented as mean \pm SEM) (all $p s>.05$ ).

Table 6

Source memory performance (mean \pm SEM).

\begin{tabular}{|c|c|c|c|c|c|c|}
\hline \multirow[t]{2}{*}{ Condition } & \multicolumn{3}{|l|}{ Male } & \multicolumn{3}{|l|}{ Female } \\
\hline & Pretest score & Posttest score & Memory decrease & Pretest score & Posttest score & Memory decrease \\
\hline Control & $0.7592 \pm 0.0266$ & $0.6641 \pm 0.0384$ & $0.0950 \pm 0.0408$ & $0.7096 \pm 0.0197$ & $0.6893 \pm 0.0313$ & $0.0204 \pm 0.0324$ \\
\hline Positive & $0.6880 \pm 0.0195$ & $0.6514 \pm 0.0356$ & $0.0366 \pm 0.0452$ & $0.7359 \pm 0.0257$ & $0.6813 \pm 0.0289$ & $0.0546 \pm 0.0348$ \\
\hline Negative & $0.6644 \pm 0.0258$ & $0.6493 \pm 0.0375$ & $0.0151 \pm 0.0357$ & $0.6822 \pm 0.0296$ & $0.6984 \pm 0.0279$ & $-0.0161 \pm 0.0346$ \\
\hline
\end{tabular}


Table 7

The number of participants in the low level and high level group.

\begin{tabular}{|c|c|c|c|c|c|c|}
\hline \multirow[t]{2}{*}{ Condition } & \multicolumn{2}{|c|}{$\begin{array}{l}\text { Arousal } \\
\text { predisposition }\end{array}$} & \multicolumn{2}{|c|}{$\begin{array}{l}\text { Emotion } \\
\text { reappraisal }\end{array}$} & \multicolumn{2}{|c|}{$\begin{array}{l}\text { Emotion } \\
\text { suppression }\end{array}$} \\
\hline & $\begin{array}{l}\text { Low } \\
\text { level }\end{array}$ & $\begin{array}{l}\text { High } \\
\text { level }\end{array}$ & $\begin{array}{l}\text { Low } \\
\text { level }\end{array}$ & $\begin{array}{l}\text { High } \\
\text { level }\end{array}$ & $\begin{array}{l}\text { Low } \\
\text { level }\end{array}$ & $\begin{array}{l}\text { High } \\
\text { level }\end{array}$ \\
\hline Control & 25 & 20 & 27 & 18 & 26 & 19 \\
\hline Positive & 26 & 22 & 23 & 25 & 25 & 23 \\
\hline Negative & 25 & 21 & 24 & 23 & 26 & 22 \\
\hline
\end{tabular}

Table 8

The range and median scores for predisposition, reappraisal and suppression.

\begin{tabular}{|c|c|c|c|c|c|c|}
\hline \multirow[t]{2}{*}{ Condition } & \multicolumn{2}{|c|}{$\begin{array}{l}\text { Arousal } \\
\text { predisposition }\end{array}$} & \multicolumn{2}{|c|}{$\begin{array}{l}\text { Emotion } \\
\text { reappraisal }\end{array}$} & \multicolumn{2}{|c|}{$\begin{array}{l}\text { Emotion } \\
\text { suppression }\end{array}$} \\
\hline & Range & Median & Range & Median & Range & Median \\
\hline Control & $24-45$ & 36 & $12-42$ & 29 & $4-21$ & 14 \\
\hline Positive & $19-54$ & 35 & $22-40$ & 31 & $5-25$ & 14 \\
\hline Negative & $27-55$ & 36 & $21-37$ & 31 & $9-24$ & 14 \\
\hline
\end{tabular}

\subsubsection{Item memory decrease as the dependent measure}

The analysis incorporating arousal predisposition showed that there were not any significant main effect of condition $(F(2,133)=$ 2.559, $\left.p=.081, \eta^{2}=.04\right)$, main effect of arousal predisposition $\left(F(1,133)=.425, p=.515, \eta^{2}=.003\right)$, or interaction $(F(2,133)=$ $\left..246, p=.782, \eta^{2}=.004\right)$.

The analysis incorporating emotion reappraisal also showed that there were not any significant main effect of condition $(F(2,134)=$ $\left.1.484, p=.23, \eta^{2}=.02\right)$, main effect of emotion reappraisal $\left(F(1,134)=.028, p=.868, \eta^{2}<.001\right)$, or interaction $(F(2,134)=$ $\left..059, p=.943, \eta^{2}=.001\right)$.

The analysis incorporating emotion suppression showed that neither the main effect of condition $(F(2,135)=1.761, p=.176$ $\left.\eta^{2}=.03\right)$ nor the main effect of emotion suppression $(F(1,135)=$ $\left..599, p=.44, \eta^{2}=.004\right)$ was significant. However, the interaction between condition and emotion suppression was significant $\left(F(2,135)=3.546, p=.032, \eta^{2}=.05\right)$, indicating that only when suppression level was low, was there a significant main effect of condition $\left(F(2,136)=4.49, p=.013, \eta^{2}=.12\right)$. Further analysis showed that when suppression level was low, participants in negative condition had better item memory consolidation than those in positive condition $(t(49)=3.23, p=.002$, Cohen's $d=.90)$. However, neither participants in the positive nor in the negative condition differed significantly from those in the control condition $(p=.224$ and $p=.292$ respectively). When the suppression level was high, no significant effect of condition was found $\left(F(2,136)=.097, p=.383, \eta^{2}=.03\right)$.

\subsubsection{Source memory decrease as the dependent measure}

The analysis with source memory decrease being the dependent measure demonstrated that there was neither main effect of condition nor main effect of any of the three emotion measures (all $p s>$.05). Hence, the results were not reported here.

\section{Discussion}

To the best of our knowledge, this is the first investigation into the effect of post-learning emotion on the effect of consolidation of both item memory and source memory. The findings are: (1) For females, post-learning negative emotion, rather than positive emotion, significantly enhanced consolidation of item memory; however, neither negative emotion nor positive emotion after learning had a significant effect on consolidation of source memory; (2) For males, neither negative nor positive emotion after learning had a significant effect on either item memory or source memory. These findings provide partial support to our hypotheses.

Although there have been a large number of studies on the effect of emotion on episodic memory (D'Argembeau \& Van der Linden, 2005; Doerksen \& Shimamura, 2001; Kensinger \& Corkin, 2003; Kensinger \& Schacter, 2006; Mather \& Nesmith, 2008), the majority of them used the to-be-remembered stimuli as the source of emotion elicitation. This kind of paradigm makes it difficult to answer the question of whether emotion has an effect on encoding or on consolidation. In our study, emotion was elicited about 10 min after the initial learning and memory was tested 30 min after the initial learning, which gave us the opportunity to examine the effect of emotion solely on consolidation of memory.

Unlike previous studies that examined the effect of post-learning emotion on consolidation of episodic memory (Nielson \& Powless, 2007; Nielson et al., 2005), we took gender differences into account. Compared with the results of Nielson et al. (2005) or Nielson and Powless (2007), we found that only for females, consolidation of item memory benefited from negative emotion elicited after learning. Such a gender difference in memory might be interpreted by the different reactions of males and females to the same emotional stimuli (Cahill et al., 2001; Canli et al., 2002; Gasbarri et al., 2007). The item memory benefit enjoyed by females can further be attributed to the modulation of amygdala via epinephrine released during emotional arousal. It has been well-established that epinephrine is released into the bloodstream in response to emotional arousal (Cahill \& McGaugh, 1998; Gold \& McCarty 1981). Although epinephrine cannot enter the blood-brain barrier, it can activate brainstem nuclei that produce norepinephrine and project it to the basolateral amygdala, outputs from which travel to other brain regions such as the hippocampus and cortex (McGaugh, 2002). Because the neurons of the basolateral amygdala tend to fire in rhythmic waves (Paré, 2003), this can cause similarly rhythmic activation in the neurons of the brain regions that receive the outputs from the basolateral amygdala. The rhythmic activation of many neurons then contributes to the long-term potentiation between the coactive neurons, thereby facilitating the memory stored in the hippocampus and cortex (Gluck, Mercado, \& Myers, 2008).

One alternative interpretation for the item memory benefit enjoyed by females in the negative condition relative to those in the positive or control conditions is that the former might do more rehearsals during the rest period until the posttest. However, across the three conditions no participant reported conducting rehearsals during the rest period. Therefore, the memory benefit might not be attributed to rehearsals.

It is indeed difficult to interpret the finding that, for females, positive emotion had no effect on consolidation of either item memory or source memory. However, it has been found in many previous studies, in which the learning stimuli itself were used as the source of emotion, that positive emotion has no effect on item memory (Dougal \& Rotello, 2007; Kapucu et al., 2008). In our study, item memory was tested 30 min after the initial learning. It is possible that the time course of item memory consolidation by positive emotion is different from that by negative emotion. It may take a longer time for the effect of positive emotion to happen than it may take for the effect of negative emotion. In addition, in our study the arousal reported by participants who watched the positive video clip was significantly greater than the arousal reported by participants who watched the negative video clip. The above explanation is more plausible considering that memory retention can be impaired by arousal when measured in the short-term (Kleinsmith \& Kaplan, 1963; Torras-Garcia, Portell-Cortes, Costa-Miserachs, \& Morgado-Bernal, 1997).

For females, a 3-min experience of negative emotion after learning significantly enhanced consolidation of item memory, but had 
no effect on consolidation of source memory. This finding adds to the existing evidence supporting the neurophysiological dissociation between item memory and source memory (Slotnick, Moo, Segal, \& Hart, 2003).

Males in both negative and positive conditions did not enjoy any memory benefit. The precise mechanism for this phenomenon is difficult to obtain, but we attempt to give the following speculation. The effect of post-learning emotion for males may be task specific. Item memory can be examined by both recall and recognition paradigms; and according to Johnson, Hashtroudi, and Lindsay (1993), there existed three types of source memory tasks. It is possible that by using other tasks the effect of post-learning emotion on males will become apparent.

Pleasure and arousal are the two dimensions of emotion (Greenwald, Cook, \& Lang, 1989; Russell, 1980). Regarding the effect of emotion on episodic memory, it is still controversial whether it is valence or arousal that plays the role. Mather (2007) claimed that arousal was the factor responsible for the effect of emotion, whereas Kensinger (2009) insisted that valence was the critical factor. In our study, although participants in the positive condition had significantly greater arousal than those in the negative and control conditions, item memory performance of participants in the positive condition did not significantly differ from those in the control condition. Considering the previous studies demonstrating the enhancement effect of arousal regardless of valence (e.g., Liu et al., 2008; Nielson \& Powless, 2007), our failure to observe a generalized effect of arousal on item memory consolidation may be due to the specific experimental conditions in our study. For example, participants were asked to remember both words and their associated font colors, whereas in many previous studies participants were required to memorize words alone. In addition, in our studies memory was tested only about $25 \mathrm{~min}$ after learning, whereas in many previous studies the interval between learning and test was a week (e.g., Nielson \& Bryant, 2005; Nielson \& Powless, 2007).

In contrast to the findings from Nielson and Lorber (2009), we did not find the effects of arousal predisposition and emotion reappraisal on consolidation of either item memory or source memory. In addition, Nielson and Lorber (2009) found that emotion suppression had no effect on memory performance; however, in our study, for participants with low level of emotion suppression, consolidation of item memory in negative condition was significantly better than that in positive condition. The difference between our results and the results from Nielson and Lorber (2009) may be explained by the difference in retention interval. In our study, memory was tested $30 \mathrm{~min}$ after the initial learning, whereas in their study the retention interval was one week. Another reason may be that in their study, the negative video clip was about oral surgery that primarily aroused disgust and even fear., whereas in our study the negative video clip mainly aroused sadness as indicated by participants' reports in the pilot study. It is possible that the modulation effects of arousal predisposition, reappraisal and suppression can vary depending on the sub-categories of negative emotion.

It is worth noting that, during the learning phase of our study, participants were instructed to memorize both the words and their font colors, that is, participants in our study had to link the learning stimuli to their peripheral information. Our paradigm is different from that in many previous studies (Liu et al., 2008; Nielson \& Lorber, 2009; Nielson \& Powless, 2007; Nielson et al., 2005), in which the learning task is only to memorize words or pictures themselves. The dual task may incur greater intra-subject variation because some participants had the ability to quickly link a word to its color by thinking up a sentence or scene based on their own life experiences, whereas others may only rely on "mechanistic" rehearsals to commit a word and its color into memory. In addition, executing such a dual task, participants in our study may experience greater stress than if they are asked to only memorize words alone. Furthermore, the attempt to associate words and their font colors may detract from attentional resources that otherwise may be completely allocated to words themselves. Another important aspect is that the colors of red and blue themselves may induce emotion (Boyatzis \& Varghese, 1993), thus leading the neutral words to be become emotionally valenced. All the above-mentioned particular features of our study may be the reasons why we obtained some results different from previous studies.

Our study has theoretical significance in several aspects. Firstly, the finding that post-learning emotion had different patterns of effects for males and females adds support to a number of studies (Cahill et al., 2001; Canli et al., 2002; Gasbarri et al., 2007) that demonstrated gender differences in responses to emotional stimuli, and points out the necessity of considering gender differences in research on the effect of emotion. Secondly, despite the large number of studies on the effect of emotion on episodic memory, none of those studies has differentiated between the effect on item memory and source memory, which are two integral elements of episodic memory. Consistent with the findings of Liu et al. (2008), our study provides the evidence that the effect of emotion on episodic memory is not universal and that it is dependent on the exact element of episodic memory. Thirdly, it is still under debate whether memory modulation by post-learning emotion depends upon the emotionality of learned stimuli (Nielson et al., 2005; Okuda, Roozendaal, \& McGaugh, 2004). Our study supports the proposition that, at least for the modulatory effect of postlearning negative emotion, learning stimuli do not have to be emotional, thereby adding support to the conclusion by Nielson et al. (2005).

Our study also has practical implications. It appears that, at least for females, a 3-min experience of negative emotion after learning contributes to the consolidation of item memory tested $30 \mathrm{~min}$ after the initial learning. Although more careful studies are needed to arrive at a decisive conclusion, it is possible that watching a short negative video clip can be used as an effective mnemonic technique. Unlike many other studies in which negative emotions such as fear or disgust were elicited, the negative video clip used in this study mainly elicited the emotion of sadness. It is reasonable to assume that, as a strategy of memory intervention, the stimuli eliciting sadness might be more desirable than the stimuli eliciting fear or disgust, although many people may prefer the stimuli eliciting pleasure.

Our study has some limitations, though. First, we only relied on participants' subjective ratings to check the effectiveness of emotion elicitation. It is possible that an arousal rating of " 8 " by one participant does not necessarily mean that he or she is more aroused that another participant who gives an arousal rating of " 6 ". In fact, in a study in which males and females viewed identical stimuli, females reported greater arousal even though the amygdala was much more strongly activated in males (see the review by Canli \& Gabrieli, 2004). Therefore, it is better that, in addition to the use of subjective reports, some objective physiological parameters such as heart rate, galvanic skin responses, or even neuroimaging activities should be recorded. The second limitation is that the examination of item memory and source memory is combined into one single task, in which participants had the opportunity to judge the source information only after they made a "hit" response, resulting in the possibility that that source memory would be constrained by item memory. The third limitation is that after watching the video participants in the control condition were asked to remain relaxed in their seats. Unlike the active control employed by previous researchers in which participants in all conditions are asked to fill in questionnaires, our resting control can lead participants to ruminate on the words they learned or 
the videos they watched, which may have influences on later memory performances.

According to the source monitoring framework by Johnson et al. (1993), there are three types of source memory tasks that respectively examine the abilities to distinguish between internal sources from external sources (e.g. between what one imagines from what one sees), to distinguish between several internal sources (e.g. between the memory of what one thought and the memory of what one said), and to distinguish between several external sources (between what one sees and what one hears). The source memory task in our study only involves the ability to distinguish two external sources. Future studies should address the question of whether the null effect of post-learning emotion observed in this study also applies to other types of source memory tasks. In our study, positive emotion induced after learning was observed to have no effect on either item memory or source memory, which were tested only $30 \mathrm{~min}$ after the initial learning. It remains to be discovered whether it takes a longer time for positive emotion than it takes for negative emotion to show the modulatory effect on the consolidation of item memory and source memory.

\section{Acknowledgments}

We extend our gratitude to Jinkuang Cheng, who offered assistance to the experiment, and to Katja Umla-Runge, who provided useful advice for the manuscript.

\section{References}

Adolphs, R., Denburg, N. L., \& Tranel, D. (2001). The amygdala's role in long-term declarative memory for gist and detail. Behavioral Neuroscience, 115, 983-992.

Anderson, L., \& Shimamura, A. P. (2005). Influences of emotion on context memory while viewing film clips. American Journal of Psychology, 118, 323-337.

Anderson, K. A., Wais, E. P., John, D. E., \& Gabrieli, D. E. J. (2006). Emotion enhances remembrance of neutral events past. Proceedings of the National Academy of Sciences of the United States of America, 103, 1599-1604.

Boyatzis, C. J., \& Varghese, R. (1993). Children's emotional associations with colors. The Journal of Genetic Psychology, 155, 77-85.

Bradley, M. M., Greenwald, M. K., Petry, M. C., \& Lang, P. J. (1992). Remembering pictures: Pleasure and arousal in memory. Journal of Experimental Psychology: Learning, Memory and Cognition, 18, 379-390.

Brown, R., \& Kulik, J. (1977). Flashbulb memories. Cognition, 5, 73-99.

Cahill, L., Haier, R. J., White, N. S., Fallon, J., Kilpatrick, L., Lawrence, C., et al. (2001) Sex-related difference in amygdala activity during emotionally influenced memory storage. Neurobiology of Learning and Memory, 75, 1-9.

Cahill, L., \& McGaugh, J. L. (1998). Mechanisms of emotional arousal and lasting declarative memory. Trends in Neurosciences, 21, 294-299.

Canli, T., Desmond, J. E., Zhao, Z., \& Gabrieli, D. E. J. (2002). Sex differences in the neural basis of emotional memories. Proceedings of the National Academy of Sciences of the United States of America, 99, 10789-10794.

Canli, T., \& Gabrieli, D. E. J. (2004). Imaging gender differences in sexual arousal. Nature Neuroscience, 7, 325-326.

Chentsova-Dutton, Y. E., \& Tsai, J. L. (2007). Gender differences in emotional response among European Americans and Hmong Americans. Cognition and Emotion, 21(1), 162-181.

Coan, J. A., \& Allen, J. B. J. (2007). Handbook of emotion elicitation and assessment. New York: Oxford University Press (p. 17).

Comblain, C., D’Argembeau, A., Van der Linden, M., \& Aldenhoff, L. (2004). The effect of ageing on the recollection of emotional and neutral pictures. Memory, 12 673-684.

Cook, G. I., Hicks, J. L., \& Marsh, R. L. (2007). Source monitoring is not always enhanced for valenced material. Memory and Cognition, 35, 222-230.

Coren, S. (1988). Prediction of insomnia from arousability predisposition scores: Scale development and cross-validation. Behaviour Research and Therapy, 26 415-420.

D’Argembeau, A., \& Van der Linden, M. (2005). Influence of emotion on memory for temporal information. Emotion, 5, 503-507.

Doerksen, S., \& Shimamura, A. P. (2001). Source memory enhancement for emotional words. Emotion, 1, 5-11.
Dougal, S., \& Rotello, C. M. (2007). Remembering emotional words is based on response bias, not recollection. Psychonomic Bulletin \& Review, 14, 423-429.

Gasbarri, A., Arnone, B., Pompili, A., Pacitti, F., Pacitti, C., \& Cahill, L. (2007). Sexrelated hemispheric lateralization of electrical potentials evoked by arousing negative stimuli. Brain Research, 1138, 178-186.

Gluck, M. A., Mercado, E., \& Myers, C. E. (2008). Learning and memory. New York: Worth Publishers (p. 405).

Gold, P. E., \& McCarty, R. (1981). Plasma catecholamines: Changes after footshock and seizure-producing frontal cortex stimulation. Behavioral and Neural Biology, $31,247-260$.

Greenwald, M. K., Cook, E. W., \& Lang, P. J. (1989). Affective judgment and psychophysiological response: Dimensional covariation in the evaluation of pictorial stimuli. Journal of Psychophysiology, 3, 51-64.

Gross, J. J., \& John, O. P. (2003). Individual differences in two emotional regulations processes: Implications for affect, relationships and well-being. Journal of Personality and Social Psychology, 85, 348-362.

Johnson, M. K., Hashtroudi, S., \& Lindsay, D. S. (1993). Source monitoring. Psychological Bulletin, 114, 3-28.

Kapucu, A., Rotello, C. M., Ready, R. E., \& Seidl, K. N. (2008). Response bias in "remembering" emotional stimuli: A new perspective on age differences. Journal of Experimental Psychology: Learning, Memory, and Cognition, 34, 703-711.

Kensinger, E. A. (2009). Remembering the details: Effects of emotion. Emotion Review, 1, 99-113.

Kensinger, E. A., \& Corkin, S. (2003). Memory enhancement for emotional words: Are emotional words more vividly remembered than neutral words? Memory $\mathcal{E}$ Cognition, 31, 1169-1180.

Kensinger, E. A., \& Schacter, D. L. (2006). Reality monitoring and memory distortion: Effects of negative, arousing content. Memory E Cognition, 34, 251-260.

Kleinsmith, L. J., \& Kaplan, S. (1963). Paired-associate learning as a function of arousal and interpolated interval. Journal of Experimental Psychology, 65 190-193.

LaBar, K. S., \& Cabeza, R. (2006). Cognitive neuroscience of emotional memory. Nature Review Neuroscience, 7, 54-64.

Liu, D. L. J., Graham, S., \& Zorawski, M. (2008). Enhanced selective memory consolidation following post-learning pleasant and aversive arousal Neurobiology of Learning and Memory, 89, 36-46.

Maddock, R. J., \& Frein, S. T. (2009). Reduced memory for the spatial and temporal context of unpleasant words. Cognition and Emotion, 23, 96-117.

Maratos, E. J., Allan, K., \& Rugg, M. D. (2000). Recognition memory for emotionally negative and neutral words: An ERP study. Neuropsychologia, 38, $1452-1465$

Mather, M. (2007). Emotional arousal and memory binding: An object-based framework. Perspectives on Psychological Science, 2, 233-252.

Mather, M., \& Nesmith, K. (2008). Arousal-enhanced location memory for pictures. Journal of Memory and Language, 58, 449-464.

McGaugh, J. (2002). Memory consolidation and the amygdala: A systems perspective. Trends in Neurosciences, 25, 456-461.

Nielson, K. A., \& Bryant, T. (2005). The effects of non-contingent extrinsic and intrinsic rewards on memory consolidation. Neurobiology of Learning and Memory, 84(1), 42-48.

Nielson, K. A., \& Lorber, W. (2009). Enhanced post-learning memory consolidation is influenced by arousal predisposition and emotion regulation but not by stimulus valence or arousal. Neurobiology of Learning and Memory. doi:10.1016/j.nlm.2009.03.002.

Nielson, K. A., \& Powless, M. (2007). Positive and negative sources of emotional arousal enhance long-term word-list retention when induced as long as thirty minutes after learning. Neurobiology of Learning and Memory, 88, 40-47.

Nielson, K. A., Yee, D., \& Erickson, K. I. (2005). Memory enhancement by a semantically unrelated emotional arousal source induced after learning. Neurobiology of Learning and Memory, 84, 49-56.

Okuda, S., Roozendaal, B., \& McGaugh, J. L. (2004). Glucocorticoid effects on object recognition memory require training-associated emotional arousal. Proceedings National Academy of Sciences of the United States of America, 101, 853-858.

Paré, D. (2003). Role of the basolateral amygdada in memory consolidation. Progress in Neurobiology, 70, 409-420.

Russell, J. A. (1980). A circumplex model of affect. Journal of Personality and Social Psychology, 39, 1161-1178.

Slotnick, S. D., Moo, L. R., Segal, J. B., \& Hart, J. Jr., (2003). Distinct prefrontal cortex activity associated with item memory and source memory for visual shapes. Cognitive Brain Research, 17, 75-82.

Torras-Garcia, M., Portell-Cortes, I., Costa-Miserachs, D., \& Morgado-Bernal, I. (1997). Long-term memory modulation by posttraining epinephrine in rats differential effects depending on the basic learning capacity. Behavioral Neuroscience, 111, 301-308.

Zorawski, M., \& Killcross, S. (2003). Glucocortoid receptor agonist enhances Pavlovian appetitive conditioning but disrupts outcome specific associations. Behavioral Neuroscience, 117, 1453-1457. 